\title{
Serial selection for invasiveness increases expression of miR-143/miR-145 in glioblastoma cell lines
}

\author{
Sunwoo Koo ${ }^{1 *}$, Gail S Martin', Kevin J Schulz', Matthew Ronck ${ }^{1}$ and L Gerard Toussaint 1,2*
}

\begin{abstract}
Background: Glioblastoma multiforme (GBM) is the most common primary central nervous system malignancy and its unique invasiveness renders it difficult to treat. This invasive phenotype, like other cellular processes, may be controlled in part by microRNAs - a class of small non-coding RNAs that act by altering the expression of targeted messenger RNAs. In this report, we demonstrate a straightforward method for creating invasive subpopulations of glioblastoma cells (IM3 cells). To understand the correlation between the expression of miRNAs and the invasion, we fully profiled 1263 miRNAs on six different cell lines and two miRNAs, miR-143 and miR-145, were selected for validation of their biological properties contributing to invasion. Further, we investigated an ensemble effect of both miR-143 and miR-145 in promoting invasion.
\end{abstract}

Methods: By repeated serial invasion through Matrige ${ }^{\circledR}$-coated membranes, we isolated highly invasive subpopulations of glioma cell lines. Phenotypic characterization of these cells included in vitro assays for proliferation, attachment, and invasion. Micro-RNA expression was compared using miRCURY arrays (Exiqon). In situ hybridization allowed visualization of the regional expression of miR-143 and miR-145 in tumor samples, and antisense probes were used investigate in vitro phenotypic changes seen with knockdown in their expression.

Results: The phenotype we created in these selected cells proved stable over multiple passages, and their microRNA expression profiles were measurably different. We found that two specific microRNAs expressed from the same genetic locus, miR-143 and miR-145, were over-expressed in our invasive subpopulations. Further, we also found that combinatorial treatment of these cells with both antisense-miRNAs (antimiR-143 and -145) will abrogated their invasion without decreasing cell attachment or proliferation.

Conclusions: To best of our knowledge, these data demonstrate for the first time that miR-143 and miR-145 regulate the invasion of glioblastoma and that miR-143 and -145 could be potential therapeutic target for antiinvasion therapies of glioblastoma patients.

Keywords: Glioblastoma, MicroRNA-143, MicroRNA-145, Invasion

\section{Background}

Glioblastoma multiforme (GBM) is the most common primary brain tumor in adults, and the discovery of this tumor in patients portends a dismal prognosis. The median survival of only $12-18$ months is due, at least in part, to its invasive phenotype - rendering complete surgical resection nearly impossible. Even more distressing

\footnotetext{
* Correspondence: swkoo@tamu.edu; gtoussaint@medicine.tamhsc.edu 'Department of Neuroscience and Experimental Therapeutics, Texas A\&M Health Science Center College of Medicine, Bryan, TX 77843, USA Full list of author information is available at the end of the article
}

to patients, family members, and caregivers is the loss of neurological function that accompanies tumor invasion, recurrence, and repeated treatments. Understanding and controlling the invasive phenotype of glioblastoma offers hope of improving therapies and preserving meaningful function.

Currently, various investigators are completing, or have recently finished, clinical trials of small molecule inhibitors in glioblastoma patients based on molecular observations of protein expression and signaling cascades (e.g. inhibitors of VEGF, TGF-beta, EGFR, m-TOR) [1].

\section{Biomed Central}


A new molecular signaling paradigm has been described in the last decade, offering more potential therapeutic targets to alter the malignant phenotype of this disease.

MicroRNAs (miRNAs) are noncoding small RNA molecules which regulate post-transcriptional gene expression and have been proposed as novel cancer biomarkers and potential targets of new anticancer therapies [2]. Several groups have reported data describing the microRNA expression profiles of glioblastma [3,4]. For example, miR-124a, $-125 a,-29 b,-7,-128$ have been reported as a glioblastma tumor suppressors while miR21 increases glioblastoma cell growth by targeting p 53 and TGF- $\beta[4,5]$. In recent years, a handful of microRNA species have been linked specifically to glioblastoma brain invasion [5-7].

Herein, we describe a simple and reproducible method for creating subpopulations of glioblastoma cells with enhanced invasive properties. We present microRNA expression data differentiating these invasive cells, and provide a rationale for investigating miR-145 and mir-143 further. Finally, we confirm the expression of miR-143 and miR-145 in invasive locations within glioblastoma samples and, via knockdown experiments, illustrate reduced invasion when their expression is abrogated.

\section{Methods}

\section{Cell lines and culture conditions}

The human glioma cell lines U87MG, U251, U373 and the rat glioma cell line $\mathrm{C} 6$ were obtained from the American Type Culture Collection (ATCC, Manassas, VA, USA). The cells were grown in Dulbecco's modified Eagle's medium (DMEM, Cellgro Media tech)) supplemented with $10 \%$ heat-inactivated fetal bovine serum, penicillin (10 IU/ $\mathrm{ml})$, and streptomycin $(10 \mathrm{ug} / \mathrm{ml})$. The cells were maintained at $37^{\circ} \mathrm{C}$ in a humidified air atmosphere at $5 \% \mathrm{CO}$.

\section{Serial selection for a sub-population of invasive cells using Boyden chambers}

For selection of invasive cells, a suspension of 300,000 tumor cells/mL in serum-free DMEM (500 ul total volume) was plated in the upper chamber of a Boydentype manifold, over a Matrigel-coated membrane (24-well insert; pore size, 8 um; BD Biosciences). DMEM medium containing $10 \%$ FBS in the lower chamber served as the chemoattractant. After incubation for $21 \mathrm{~h}$ at $37^{\circ} \mathrm{C}$, those non-invading cells from the upper surface of the membrane were scrubbed off with cotton swabs. The cells that invaded to the bottom surface of the insert were trypsinized and seeded into flasks containing DMEM supplemented with $10 \%$ FBS. These cells were propagated and went through the above selection procedure twice more. The cells that were collected after the third selection procedure were propagated resulting in the invasive subpopulation cell line termed IM3.

\section{Boyden chamber invasion assay}

For migration assays, a suspension of 300,000 tumor cells/ $\mathrm{ml}$ in serum-free DMEM (500 ul total volume) was plated in the upper chamber of a Matrigel-coated membrane insert (24-well insert; pore size, 8 um; BD Biosciences). DMEM medium containing 10\% FBS in the lower chamber served as the chemoattractant. The cells were incubated at $37^{\circ} \mathrm{C}$ for various times depending on our laboratory experience regarding their relative speeds of invasion (18 hrs for U87 and U251; 36 hrs for U373; $17 \mathrm{hrs}$ for C6). The non-invading cells were removed with cotton swabs. Those cells that had migrated to the lower side of the membrane were fixed and stained with hematoxylin. The invading cells on quadruplicate membranes were quantitated by counting across a diameter of each memebrane under 40X microscopic magnification.

\section{Cell attachment assay}

For the cell attachment assay, wells of a 24-well tissue culture plate were coated with Matrigel Basement Membrane Matrix (BD Biosciences) diluted 1:10 with serum-free medium for $2 \mathrm{~h}$ at room temperature. The unbound material was aspirated and the wells were rinsed with serumfree medium. Cells were detached with trypsin/EDTA and rinsed in serum free medium. Approximately 20,000 cells/ $\mathrm{ml}$ in $10 \%$ DMEM were plated into the wells, and the plate was incubated at $37^{\circ} \mathrm{C}$ for $4 \mathrm{~h}$. Wells were gently washed with PBS and the attached cells were fixed in $4 \%$ paraformaldehyde and stained with hematoxylin QS (Vector Laboratories) for $2 \mathrm{~min}$. Wells were washed again in PBS and the stained cells were counted.

\section{Proliferation assay}

For each cell line, parental and IM3 cells were plated at a density of 5000 cells per well, each in 8 wells of 96-well plates containing $10 \% \mathrm{FBS} / \mathrm{DMEM}$ and maintained at $37^{\circ} \mathrm{C}$. The rate of cell proliferation was determined using CellTiter $96 \mathrm{AQ}_{\text {ueous }}$ One Solution (Promega, Madison, WI) at 4, 24, 48, 72 and 96 hours after plating. An MTS assay was performed by following the protocol previously reported [8]. Eight media only containing wells were used to normalize the absorbance values of the wells containing cells. Growth curves were generated with normalized absorbance compared to the 4-hour data, at 24, 36, 48, and 72 hours.

\section{RNA isolation}

Total cellular RNA was extracted using the mirVana miRNA Isolation kit (Ambion) per manufacturer's instructions for total RNA isolation. The RNA concentration was determined by spectrophotometric absorbance at $260 \mathrm{~nm}$, and the quality of the RNA was determined by Agilent 2100 Bioanlayzer instrument (Agilent Technologies, Santa Clara, CA). All RNA samples showed 
high quality (RNA integrity number (RIN) > 9.0) and were without RNA degradation or DNA contamination.

\section{Real-time quantitative RT-PCR analysis}

qRT-PCR was performed in triplicate by using LNA ${ }^{\mathrm{TM}}$ PCR primer sets (miR-143, -145 and U6), SYBR ${ }^{\circledR}$ Green PCR kit, and the ViiA ${ }^{\mathrm{TM}} 7$ real-time PCR system (Exiqon). Total RNA extracted from U87, U251, U373, and their corresponding invasive subpopulations, was used for cDNA synthesis (Exiqon). The PCR reaction was started with cDNA $(140 \mathrm{ng} / \mu \mathrm{l})$ in $25 \mu \mathrm{l}$ (total) assay mix. The PCR cycle settings were those recommended by Exiqon: $95^{\circ} \mathrm{C}, 10 \mathrm{~min} ; 40$ amplification cycle at $95^{\circ} \mathrm{C}(10 \mathrm{~s})$ and $65^{\circ} \mathrm{C}(1 \mathrm{~min}), 1.6^{\circ} \mathrm{C} / \mathrm{s}$ ramp-rate.

\section{Knockdown miRNA transfection}

Parental and IM-3 cells from each of three cell lines (U87, U251, U373) cells were transfected with either fluoresceinlabeled locked nucleic acid (LNA) antagomirs targeting miR-143 and miR-145 (Exiqon, Vedbaek, Denmark) at a final concentration of $50 \mathrm{nM}$ (each probe) using Lipofectamine 2000 per manufacturer's instructions (Invitrogen, Carlsbad, CA). Control cells were transfected with an untargeted antagomir at a final concentration of $100 \mathrm{nM}$. Transfection medium was changed 8 hours after transfection and replaced with Optimem medium (Invitrogen Carlsbad, CA) for overnight recovery. The efficiency of the transfection was assessed with fluorescence microscope. A Double knockdown transfection of U87 and U87 IM3 cells using fluorescein-labeled miR-143 and miR-145 knockdown probe at final concentration of $50 \mathrm{nM}$ each and control LNA knockdown probe at final concentration of $100 \mathrm{nM}$ was performed using Lipofectamine 2000 per manufacturer's instructions. Once again, culture medium was changed 8 hours after transfection and replaced with Optimem medium (Invitrogen Carlsbad, CA) for overnight recovery and the efficiency of the transfection was assessed with fluorescence microscopy. Antisense micro RNAs (antimir-143 and -145) for knockdown were purchased from Exiqon.

\section{Boyden chamber migration assay of knockdown miRNA Transfected cells}

A suspension of 300,000 cells $/ \mathrm{mL}$ in serum-free medium of parental and IM3 cells transfected with LNA antagomirs (against miR-143 and miR-145 or a control LNA antagomir) was plated in the upper chamber of a Boyden manifold over a Matrigel-coated membrane (24-well insert; pore size, 8 um; BD Biosciences). DMEM medium containing $10 \%$ FBS in the lower chamber served as the chemoattractant. The cells were incubated for 17-36 hours at $37^{\circ} \mathrm{C}$. The non-invading cells were removed with cotton swabs. Those cells that had migrated to the lower side of the membrane were fixed and stained with DAPI. The invading cells of quadruplicate membranes were counted under the microscope at $40 \times$ magnification.

\section{In situ detection of miRNAs}

In situ detection of miRNAs was performed on 8-10-um frozen tissue sections from xenografts of human GBM tumors in mouse brain. Sections were fixed using fresh ice-cold $4 \%$ paraformaldehyde for $1 \mathrm{~h}$, acetylated in acetic anhydride/triethanolamine, and prehybridized in hybridization solution ( $50 \%$ formamide, $5 \mathrm{X} \mathrm{SSC}, 0.5 \mathrm{mg} / \mathrm{mL}$ yeast tRNA, 1X Denhardt's solution) at $25^{\circ} \mathrm{C}$ below the predicted $\mathrm{T}_{\mathrm{m}}$ value of the LNA probe for $30 \mathrm{~min}$. Probes (4 pmol; fluorescein isothiocyanate (FITC)-labeled LNAmodified oligonucleotide; Exiqon) complementary to the mature miRNA (miR-143 and miR-145) were hybridized to the sections for $2 \mathrm{~h}$ at $25^{\circ} \mathrm{C}$ lower than predicted $\mathrm{T}_{\mathrm{m}}$ value of the LNA probe. Post-hybridizations washes were performed in $0.5 \mathrm{X} \mathrm{SSC}$ at $8^{\circ} \mathrm{C}$ above the hybridization temperature and the in situ hybridization signal was detected by incubation with horseradish peroxidase (HRP)-conjugated anti-FITC. The signal was then amplified using FITC-conjugated tyramide (Tyramide Signal Amplification Plus Kit, Fluorescein, Perkin-Elmer) according to the manufacturer's instructions. Slides were mounted in Vectashield Hard Set mounting medium containing 4',6-diamidino-2-phenylindole (DAPI) (Vectashield mounting medium with DAPI, Vector Laboratories) and analyzed with an Olympus CKX41 microscope equipped with a CCD camera and Olympus software.

\section{Statistical analysis}

Data are presented as mean $+/$ - standard deviation. For parental and IM-3 comparisons, the Student's $t$ test (twotailed) was used to determine statistical significance. A student's $t$ test with a value of $P<0.05$ was considered significant.

\section{Results}

Creation and characterization of highly invasive glioblastoma cell line subpopulations

Serial selection for invasion through Matrigel ${ }^{\mathbb{R}}$-coated Boyden chamber membranes is a viable tool to separate glioblastoma cell lines into parental and highly-invasive sub-populations (IM3 cells). Confirmatory assays revealed 15-, 5-, 20- and 1.5-fold (U87, U251, U373 and C6 cells lines) increases in the number of invading cells when comparing selected IM3 populations to their parental counterparts (Figure 1B, 2A, Additional file 1A). This phenotypic alteration has been consistent through multiple (up to 20) passages, and at least 3 freeze-thaw cycles. Possible confounders for Boyden chamber invasion data were investigated. Enhanced attachment to Matrigel ${ }^{\mathbb{B}}$ or an increase in cellular proliferation could complicate the interpretation of invasion results. We 


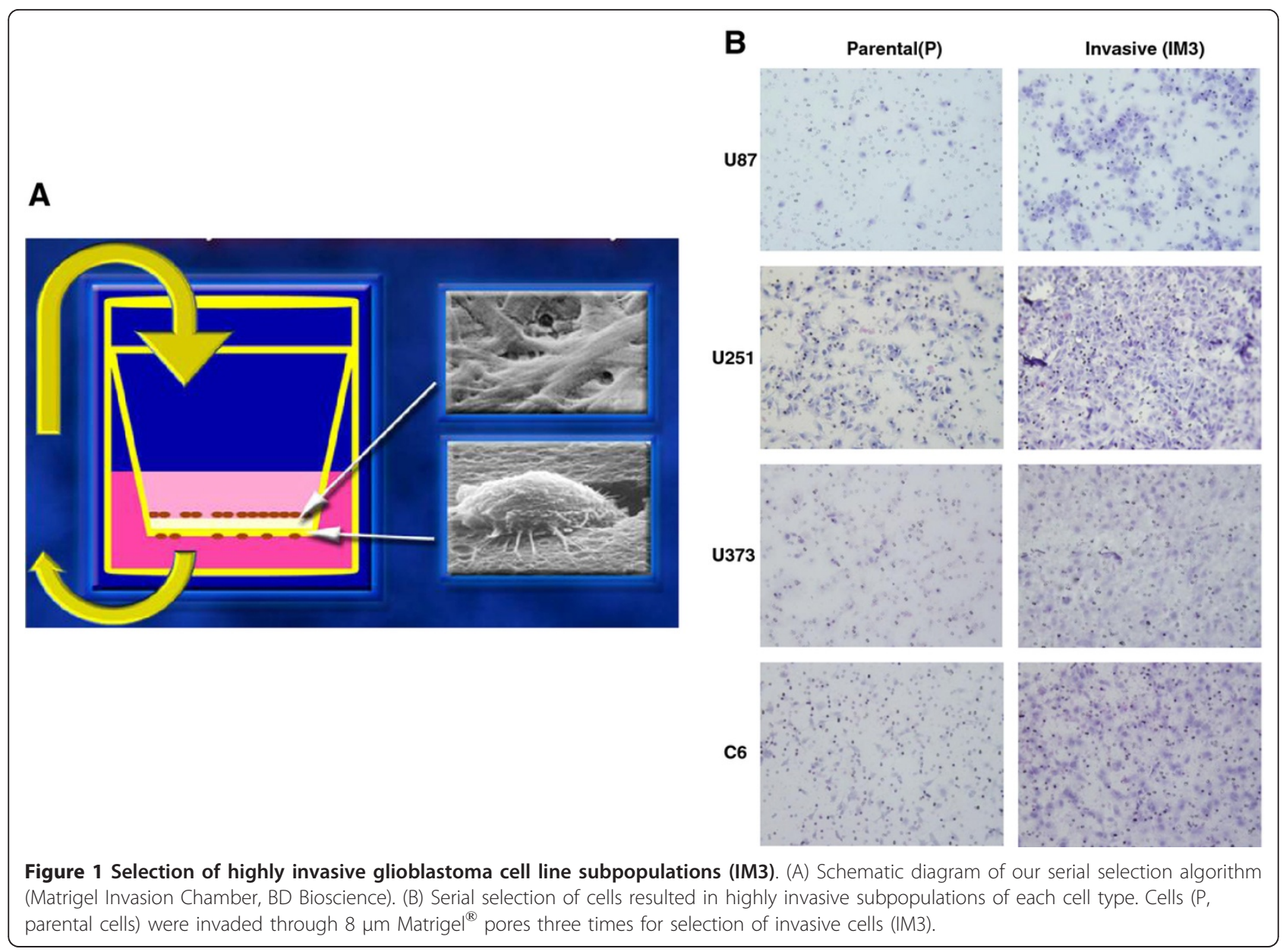

investigated both, and found no significant differences in these assays between parental and IM3 cell lines (Figure 2B, C, Additional file 1B). Serial selection resulted in a stable and predictable phenotype.

\section{Both miR-145 and miR-143 are expressed at a high level in IM3 cell lines}

Using the human miRCURY LNA microarray platform from Exiqon, we analyzed expression of all miRBASE v.16 human microRNAs, and compared data between parental and IM3 cells (Figure 3). The resulting list of preferentially expressed miRNAs was filtered in the following manner: 1) probeset data was collected when 6 data points were nontrivial - parental and IM3 subpopulations of all 3 human lines each produced adequate RNA hybridization for data above background, 2) the resulting fold change data was sorted according to highest observed fold-change, 3 ) the top 38 miRNAs, all with at least one fold change $>2$, were analyzed looking for the direction of change between parental lines and IM-3 subpopulations to be similar between U87, U251, and U373 data. That is, we were interested in miRNAs that either moved uniformly up or down in invasive subpopulations. We found that miR-143 and -145 were over-expressed in all three IM3 cell lines compared to their parental counterparts (Figure 3B, red arrow). The expression level of miR-143 in IM3 cells is 2.5, 1.5 and 7.5 times higher than that of parental cells, while miR145 was also overexpressed by 2, 1.2 and 4 fold (U87, $\mathrm{U} 251$ and $\mathrm{U} 373$ respectively) (Figure $3 \mathrm{~B}$ ). The parallel movement in expression between these molecules across cell lines was striking (Figure 3A vs. 3B). We also performed qRT-PCR for quantitation and validation of microarray results. As expected, the expression of these miRNAs differed significantly between parental and IM3 cells: $\sim 3$ fold change of both miR-143 and -145 in U87, 2 fold change of both miR-143 and -145 in $\mathrm{U} 251, \sim 4$ fold and $\sim 3$ fold change of miR-143 and -145 respectively in $\mathrm{U} 373$.

\section{The invasion of human GBM cells was down-regulated} after treatment with antisense miR-143 and -145

To confirm the role of miR-143 and -145 in enhanced invasiveness of IM3 cells, we tested the efficacy of combinatorial transfection of antisense LNA probes targeting human miR-143 and -145 (antagoMIR ${ }^{\circledR}$ Exiqon) 


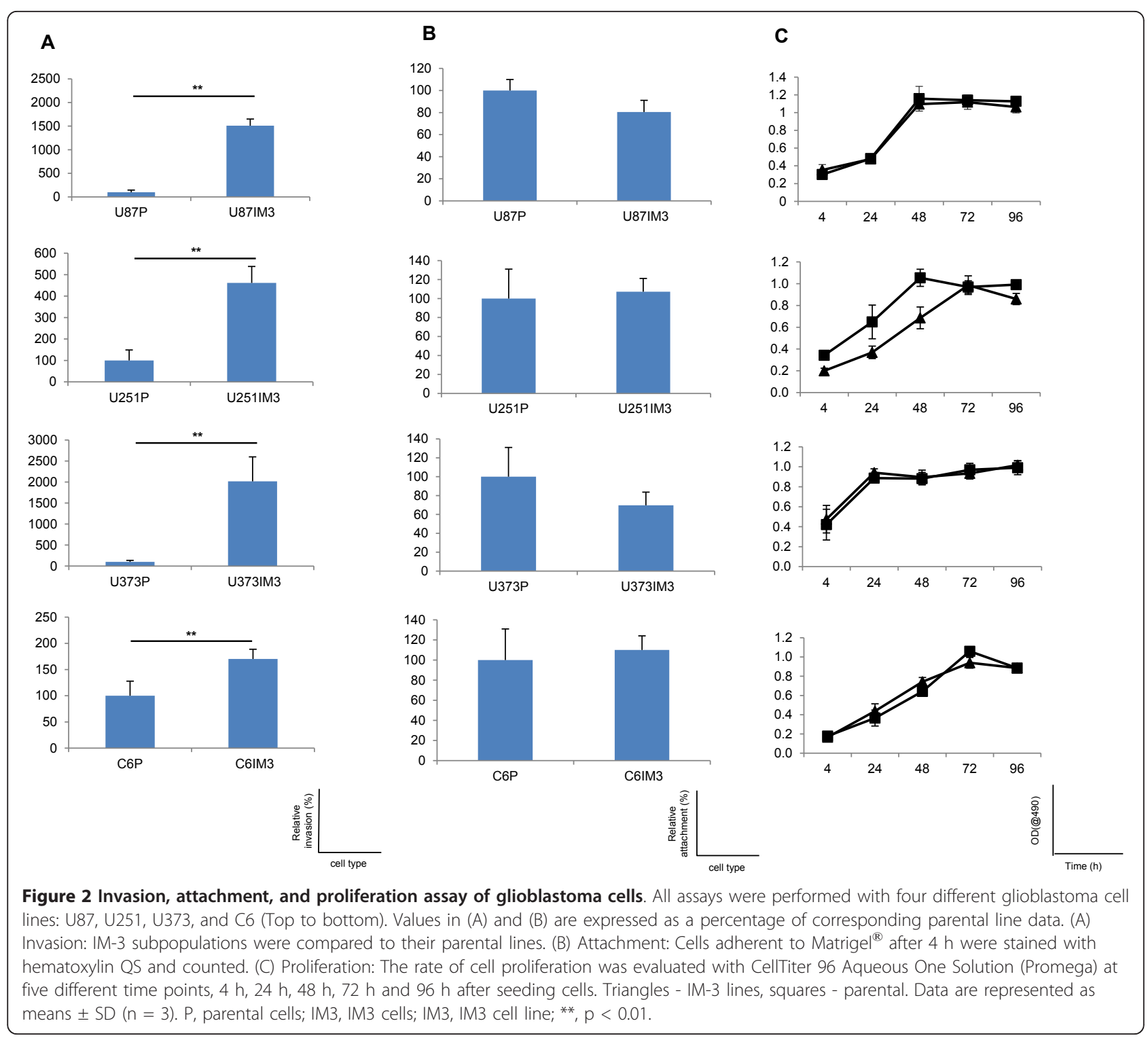

compared to untargeted/scrambled LNA probes. Double-treatment with antisense probes against miR-143 and -145 caused a decrease in invasion within the IM3 subpopulations (Figure 4A-C). The anti-invasive effect of these antimiRs is similar across all three human glioma cell lines: reducing invasion counts by 40 to $50 \%$ (Figure 4A-C). There was, however, a less predictable effect of treatment with either antimiR-143 or -145 alone (data not shown). These results suggest that double treatment with antimiR-143 and -145 has a synergistic effect in limiting the invasion of glioblastoma cells.

\section{Human glioblastoma expresses miR-143 and miR-145 in typical areas of invasion}

In-situ hybridization confirmed the expression of miR143 and miR-145 in human samples of glioblastoma. In freshly resected tumor, the expression appears prominently in the perivascular space, while tumors xenografted orthotopically into nude mice express these molecules near the tumor-brain interface 5 .

\section{Discussion}

In this study, we test the hypothesis that microRNAs are indeed key regulators of the invasive phenotype of glioblastoma. We propose that two specific micro-RNAs, miR-143 and miR-145, expressed from the same genetic locus [9], act in concert to promote glioma invasion. In contrast, miR-143 and miR-145 have been described by others as a tumor suppressor molecules in most nonneural human cancer cell lines tested $[10,11]$. Fewer reports suggest a tumor type-specific difference in their effect, and an oncogenic role in certain settings [12]. 


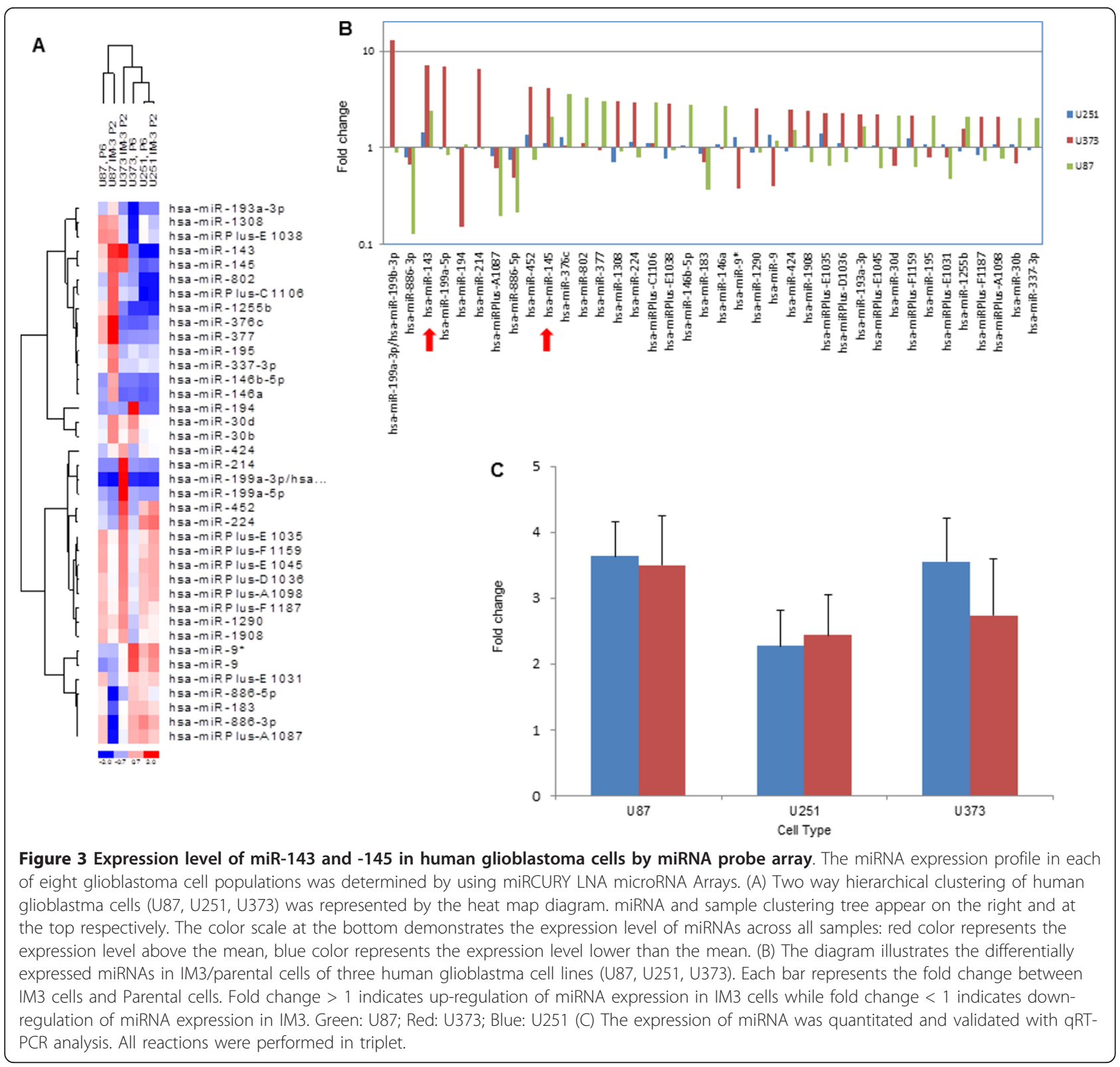

Finally, Cordes et al. suggest a role for miR-143/145 in promoting the differentiation of neural crest cells into vascular smooth muscle [9]. Future investigations will help define whether there is a causal connection between miR143 and miR-145 promoting invasion and decreasing tumor growth or, alternatively, promoting more mesenchymal behavior.

\section{Serial selected invasive cell line (IM3)}

The process of glioblastoma invasion into surrounding parenchyma is complex - involving cell attachment, cytoskeletal remodeling, membrane deformation, extracellular matrix proteolysis, detachment, and altered metabolic demands [13-15]. One model for examining the myriad of genetic and epigenetic alterations necessary for invasion was created in our laboratory via serial selection through Matrigel ${ }^{\circledR}$ in a modified Boyden chamber [16]. Invasion towards a serum gradient, trypsinization, regrowth, and further iterative invasion proved to be a reproducible method for selecting invasive glioma cells. Further, the invasive phenotype of selected cells has remained constant through multiple passages (up to 20) and through freeze-thaw cycles (up to 3 tested). Even in the C6 rat glioma cell line, the most invasive of the four lines tested in these experiments, we were able to create a phenotypically distinct subpopulation. 

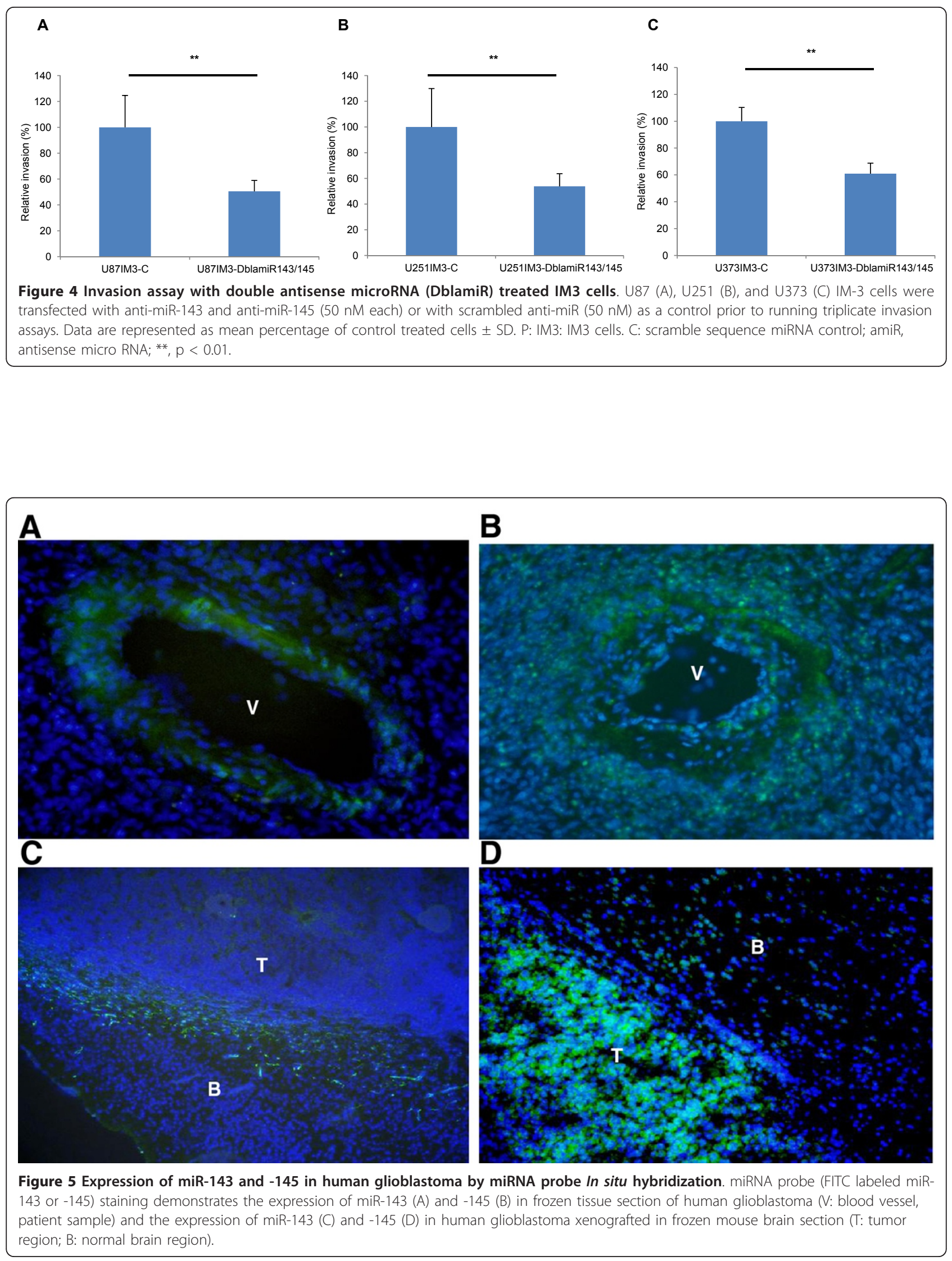


\section{Overexpression of miR-143 and miR-145 in glioblastoma cells}

From our collection of cell lines and their more invasive sub-populations, we extracted RNA and generated miRNA expression data by hybridization to a popular microarray platform [17]. Accordingly, we were interested in miRNAs whose expression was increased in all the invasive subpopulations compared to their parental lines, or those miRNAs whose expression was uniformly decreased in invasive cells. Data used to make this decision was derived from the three human glioma lines (U87, U373, U251), all using the same human-specific Exiqon expression array platform. Although we had no a priori knowledge of the genetic loci involved, we noticed a significant parallel in the pattern of upregulation of miR-143 and miR-145 across the three human lines (Figure 3B). Investigation of the chromosomal location of these miRNAs confirmed a reasonable explanation for the parallel expression - they are encoded in the same transcript $[9,18]$.

\section{Expression of miR-143 and miR-145 in resected human glioblastoma samples}

Owing to its unique matrix composition, the use of Matrigel ${ }^{\circledR}$ for selection of invasive glioma cells may bias our results towards identification of mediators of motility along basement membranes [19]. Yet, the propensity of glioblastoma to invade into these spaces: the perivascular Virchow-Robin space and the subpial plane, has been recognized since Scherer published landmark papers [20]. In our hands, in situ hybridization confirmed the expression of miR-143 and miR-145 along the perivascular space in frozen samples of resected human glioblastoma (Figure 5A-D). The downstream effects of the expressing the miR-143/-145 locus may allow for enhanced mobility along the unique extracellular matrix outside cerebral vasculature. In xenograft samples of glioblastoma, grown in the brains of nude mice, we found enhanced expression of miR-143 and miR-145 on either side of the tumor-host brain interface (Figure 5A, B). Although a typical basement membrane matrix has not been described at the interface between xenograft tumor and host brain, reactive astrocytes (fibronectin), and the invading tumor itself (vitronectin, proteoglycans) can both secrete ECM components shared by the Matrigel ${ }^{\circledR}$ substrate $[21,22]$. Our selection of cells capable of invading through Matrigel ${ }^{\circledR}$ may have identified those with cellular machinery allowing growth and invasion along the Virchow-Robin space and the brain-tumor interface. In situ regional expression of our target microRNAs in tumor samples illustrates, once again, the regional heterogeneity of glioblastoma, and supports our hypothesis about their role in mediating invasion.

\section{Synergistic anti-invasive effect of knocking down both of miR-143 and miR-145}

In a series of transient transfection experiments, we have shown that antisense microRNAs treatment knocking down miR-143 and miR -145, in combination, abrogates the invasive phenotype of each of our human glioma cell lines (Figure 4A-C). Interestingly, in the U87 cell line, miR-145 knockdown alone was sufficient to produce a reduction in invasive phenotype (Data not shown), while in other cell lines, the combined treatment was needed to see the same effect. This finding underscores the necessity to characterize the expression of possible downstream targets across multiple human glioblastoma lines and primary cultures, and to find those characteristic differences between lines that account for the inconsistencies. More importantly, this points towards a molecular synergy that is not yet defined.

\section{Potential targets of miR-143/miR-145 and future directions}

In an effort to characterize the important downstream targets of miR-143 and miR-145, we utilized three algorithms to score the likelihood of each to hybridize to, and affect translation of, particular genes. The on-line search algorithms TargetScan, PicTar, and MicroCosm were all used to stratify targets. By filtering the resulting lists for those targets that were identified by multiple search algorithms, we found a number of potential miR-145 targets (especially srGAP1) in the Slit/Robo pathway, which was recently recognized as an inhibitor of brain tumor cellular migration and invasion $[23,24]$. In western-blot experiments, we recognized a decreased expression of srGAP1 in the invasive subpopulation of U87 cells. Further definition of the role of srGAP1 activity, along with clearer understanding of other downstream mediators of both miR-143 and miR-145 is necessary.

The relationship between miR-143/miR-145 expression and other known mediators of glioma invasion must be defined. Very recently, Yan et al. described the likely micro-RNA regulators of MMP-9 expression - a molecule with known importance in survival and invasion of glioblastoma [25]. According to their results, 14 miRNAs (including miR-143, miR-210 and miR-214) positively regulate the overexpression of MMP-9 in glioma cell lines we tested (U87 and U251). Their results support our contention that miR-143 mediates invasion. Further, on review of our expression data, we also find miR-210 and miR-214 are upregulated in our IM-3 lines compared to their parental controls. Further studies are needed to identify the correlation between the expression of microRNAs and matrix metalloproteases in glioblastoma.

The identification of miR-143 and miR-145 as positive regulators of glioblastoma invasion is novel. In fact, most authors have described these molecules as tumor 
suppressors and mediators of differentiation into vascular smooth muscle $[9,12,18]$. A single report supports the role of miR-145 as an oncogene in metastatic colorectal cancer cells $[12,26]$. However, hypotheses based on data generated from malignancies outside the central nervous system often prove unsupported in glioblastoma. The behavior of this tumor is unique - it is markedly invasive in the host organ yet metastases are almost non-existent. The tumor grows quickly, but invasive cells are often slower-growing [27]. Emerging data from our laboratory and those of other investigators support a role for the miR-143/145 locus in promoting glioma invasion.

\section{Conclusions}

The micro-RNA mediators of glioblastoma invasion are incompletely defined. We present, in this publication, a method for creating stable and invasive subcultures of common glioma cell lines, and we use them to define the micro-RNA regulators of invasion. Two molecules of interest, miR-143 and miR-145, are likely key proinvasive mediators, and our data correlate well with those emerging from other investigators. Understanding the unique pathophysiology of glioblastoma invasion will help direct future drug design and therapies aimed at prolonging meaningful quality of life.

\section{Additional material}

Additional file 1: The invasion and attachment raw plots. Invasion assay (A) and attachment assay (B) data from glioblastoma cell lines: U87, U251, U373, C6. (blue rectangles) Parental (red rectangles) IM3.

\section{Acknowledgements and funding}

This work was supported by St. Joseph Regional Health Center Research Grant.

\section{Author details}

${ }^{1}$ Department of Neuroscience and Experimental Therapeutics, Texas A\&M Health Science Center College of Medicine, Bryan, TX 77843, USA. ${ }^{2}$ The Texas Brain and Spine Institute, Bryan, TX 77807, USA.

\section{Authors' contributions \\ LGT performed the initial project design, the experimental design, and edited the manuscript and data analysis. SWK participated in the experiment, performed data analysis, and wrote the initial manuscript. GM was a key developer of the experimental approach, KS performed and interpreted experiments, and MR designed the methodology for miRNA target analysis. All authors read and approved the final manuscript.}

\section{Competing interests}

The authors declare that they have no competing interests.

Received: 27 October 2011 Accepted: 10 April 2012

Published: 10 April 2012

\section{References}

1. Huang TT, Sarkaria SM, Cloughesy TF, Mischel PS: Targeted therapy for malignant glioma patients: lessons learned and the road ahead. Neurotherapeutics 2009, 6(3):500-512.
2. Esquela-Kerscher A, Slack FJ: Oncomirs - microRNAs with a role in cancer. Nat Rev Cancer 2006, 6(4):259-269.

3. Yan W, Zhang W, Sun L, Liu Y, You G, Wang Y, Kang C, You Y, Jiang T: Identification of MMP-9 specific microRNA expression profile as potential targets of anti-invasion therapy in glioblastoma multiforme. Brain Res 2011, 1411:108-115.

4. Novakova J, Slaby O, Vyzula R, Michalek J: MicroRNA involvement in glioblastoma pathogenesis. Biochem Biophys Res Commun 2009, 386(1):1-5.

5. Papagiannakopoulos T, Shapiro A, Kosik KS: MicroRNA-21 targets a network of key tumor-suppressive pathways in glioblastoma cells. Cancer Res 2008, 68(19):8164-8172.

6. Chan JA, Krichevsky AM, Kosik KS: MicroRNA-21 is an antiapoptotic factor in human glioblastoma cells. Cancer Res 2005, 65(14):6029-6033.

7. Gabriely G, Wurdinger T, Kesari S, Esau CC, Burchard J, Linsley PS, Krichevsky AM: MicroRNA 21 promotes glioma invasion by targeting matrix metalloproteinase regulators. Mol Cell Biol 2008, 28(17):5369-5380.

8. Jiang Q, Wei H, Tian Z: Poly I:C enhances cycloheximide-induced apoptosis of tumor cells through TLR3 pathway. BMC Cancer 2008, 8(1):12.

9. Cordes KR, Sheehy NT, White MP, Berry EC, Morton SU, Muth AN, Lee TH, Miano JM, Ivey KN, Srivastava D: miR-145 and miR-143 regulate smooth muscle cell fate and plasticity. Nature 2009, 460(7256):705-710.

10. Akao Y, Nakagawa Y, Naoe T: MicroRNAs 143 and 145 are possible common onco-microRNAs in human cancers. Oncol Rep 2006, 16(4):845-850.

11. Kitade $Y$, Akao $Y$ : MicroRNAs and their therapeutic potential for human diseases: microRNAs, miR-143 and -145, function as anti-oncomirs and the application of chemically modified miR-143 as an anti-cancer drug. J Pharmacol Sci 2010, 114(3):276-280.

12. Arndt GM, Dossey L, Cullen LM, Lai A, Druker R, Eisbacher M, Zhang C, Tran N, Fan H, Retzlaff K, et al: Characterization of global microRNA expression reveals oncogenic potential of miR-145 in metastatic colorectal cancer. BMC Cancer 2009, 9:374.

13. Yamamoto $M$, Ueno $Y$, Hayashi S, Fukushima T: The role of proteolysis in tumor invasiveness in glioblastoma and metastatic brain tumors. Anticancer Res 2002, 22(6C):4265-4268

14. Grobben B, De Deyn PP, Slegers H: Rat C6 glioma as experimental model system for the study of glioblastoma growth and invasion. Cell Tissue Res 2002, 310(3):257-270

15. Ulrich TA, de Juan Pardo EM, Kumar S: The mechanical rigidity of the extracellular matrix regulates the structure, motility, and proliferation of glioma cells. Cancer Res 2009, 69(10):4167-4174.

16. Fong CJ, Sutkowski DM, Kozlowski JM, Lee C: Utilization of the Boyden chamber to further characterize in vitro migration and invasion of benign and malignant human prostatic epithelial cells. Invasion Metastasis 1992, 12(5-6):264-274.

17. Sokilde R, Kaczkowski B, Podolska A, Cirera S, Gorodkin J, Moller S, Litman T: Global microRNA analysis of the $\mathrm{NCl}-60$ cancer cell panel. $\mathrm{Mol}$ Cancer Ther 2011, 10(3):375-384

18. Akao Y, Nakagawa Y, Hirata I, lio A, Itoh T, Kojima K, Nakashima R, Kitade Y, Naoe T: Role of anti-oncomirs miR-143 and -145 in human colorectal tumors. Cancer Gene Ther 2010, 17(6):398-408.

19. Paulus W, Tonn JC: Basement membrane invasion of glioma cells mediated by integrin receptors. J Neurosurg 1994, 80(3):515-519.

20. Peiffer J, Kleihues P: Hans-Joachim Scherer (1906-1945), pioneer in glioma research. Brain Pathol 1999, 9(2):241-245.

21. Rempel SA, Golembieski WA, Ge S, Lemke N, Elisevich K, Mikkelsen T, Gutierrez JA: SPARC: a signal of astrocytic neoplastic transformation and reactive response in human primary and xenograft gliomas. $J$ Neuropathol Exp Neurol 1998, 57(12):1112-1121.

22. Giese A, Loo MA, Rief MD, Tran N, Berens ME: Substrates for astrocytoma invasion. Neurosurgery 1995, 37(2):294-301, discussion 301-292.

23. Mertsch S, Schmitz N, Jeibmann A, Geng J-G, Paulus W, Senner V: Slit2 involvement in glioma cell migration is mediated by Robo1 receptor. $J$ Neurooncol 2008, 87(1):1-7.

24. Werbowetski-Ogilvie TE, Seyed Sadr M, Jabado N, Angers-Loustau A, Agar NY, Wu J, Bjerkvig R, Antel JP, Faury D, Rao Y, et al: Inhibition of medulloblastoma cell invasion by Slit. Oncogene 2006, 25(37):5103-5112.

25. Yan W, Zhang W, Sun L, Liu Y, You G, Wang Y, Kang C, You Y, Jiang T: Identification of MMP-9 specific microRNA expression profile as 
potential targets of anti-invasion therapy in glioblastoma multiforme. Brain Res 2011, 1411:108-115.

26. Sachdeva M, Mo YY: miR-145-mediated suppression of cell growth, invasion and metastasis. Am J Transl Res 2010, 2(2):170-180.

27. Demuth T, Berens ME: Molecular mechanisms of glioma cell migration and invasion. J Neurooncol 2004, 70(2):217-228.

\section{Pre-publication history}

The pre-publication history for this paper can be accessed here: http://www.biomedcentral.com/1471-2407/12/143/prepub

doi:10.1186/1471-2407-12-143

Cite this article as: Koo et al:: Serial selection for invasiveness increases expression of miR-143/miR-145 in glioblastoma cell lines. BMC Cancer 2012 12:143.

Submit your next manuscript to BioMed Central and take full advantage of:

- Convenient online submission

- Thorough peer review

- No space constraints or color figure charges

- Immediate publication on acceptance

- Inclusion in PubMed, CAS, Scopus and Google Scholar

- Research which is freely available for redistribution 